\title{
Uniformly Distributed Graphene Domain Grows on Standing Copper via Low-Pressure Chemical Vapor Deposition
}

\author{
Shih-Hao Chan, ${ }^{1}$ Sheng-Hui Chen, ${ }^{1}$ Wei-Ting Lin, ${ }^{1}$ and Chien-Cheng Kuo ${ }^{1,2}$ \\ ${ }^{1}$ Department of Optics and Photonics, National Central University, 300 Chung-Da Road, Chung-Li 32001, Taiwan \\ ${ }^{2}$ Graduate Institute of Energy Engineering, National Central University, 300 Chung-Da Road, Chung-Li 32001, Taiwan \\ Correspondence should be addressed to Chien-Cheng Kuo; cckuo@ncu.edu.tw
}

Received 28 May 2013; Accepted 1 August 2013

Academic Editor: Ying Liu

Copyright (c) 2013 Shih-Hao Chan et al. This is an open access article distributed under the Creative Commons Attribution License, which permits unrestricted use, distribution, and reproduction in any medium, provided the original work is properly cited.

\begin{abstract}
Uniformly distributed graphene domains were synthesized on standing copper foil by a low-pressure chemical vapor deposition system. This method improved the distribution of the graphene domains at different positions on the same piece of copper foil along the forward direction of the gas flow. Scanning electron microscopy (SEM) showed the average size of the graphene domains to be about $\sim 20 \mu \mathrm{m}$. This results show that the sheet resistance of monolayer graphene on a polyethylene terephthalate (PET) substrate is about $\sim 359 \Omega / \square$ whereas that of the four-layer graphene films is about $\sim 178 \Omega / \square$, with a transmittance value of $88.86 \%$ at the $550 \mathrm{~nm}$ wavelength. Furthermore, the sheet resistance can be reduced with the addition of $\mathrm{HNO}_{3}$ resulting in a value of $84 \Omega / \square$. These values meet the absolute standard for touch sensor applications, so we believe that this method can be a candidate for some transparent conductive electrode applications.
\end{abstract}

\section{Introduction}

Tin oxide is a wide bandgap semiconductor with a low ntype resistivity $\left(\sim 10^{-3} \Omega \mathrm{cm}\right)$ and high transparency $(\sim 90 \%)$ in the visible light region. Up to now, indium tin oxide (ITO) has been the most common choice for many devices such as solar cells [1], light emitting diodes [2], and touch panels [3]. However, ITO is unlikely to continue to be utilized to meet future needs due to its high cost, scarcity, and irreversible loss in conductivity on bending [4]. Zinc oxide ( $\mathrm{ZnO})$ based thin films have attracted interest as conductive oxide films because of their low cost and abundance of the elements. They are also nontoxic and easy to fabricate [5]. However, as with ITO, the stretching of zinc oxide based films also leads to irreversible loss in conductivity on bending. The unique properties of graphene, a two-dimensional material packed into a honeycomb lattice of $\mathrm{sp}^{2}$ carbon atoms, have attracted much attention in recent years [6]. In comparison to ITO and $\mathrm{ZnO}$ thin films, graphene films have high transmittance in the visible region, high mechanical strength, are chemically stable, and, last but not least, are flexible [7].
Production of high quality, uniform grain size and large area graphene films is necessary for electrode applications. Very recently, $\mathrm{Li}$ et al. demonstrated a transfer technique for the stacking of four-layer graphene films which showed a sheet resistance and transmittance of $350 \Omega / \square$ and $~ 90 \%$, respectively [8]. Bae et al. reported layer-by-layer stacking for the fabrication of a $\mathrm{HNO}_{3}$ doped four-layer graphene film with a measured sheet resistance value as low as $\sim 30 \Omega / \square$ at $\sim 90 \%$ transparency [9]. Most importantly, the distribution of the graphene domains is not equal at the different positions on the same copper foil along the forward direction of the gas flow [10-12]. Zhang et al. studied the synthesis of large area, uniform graphene films on tilted copper foil against gas flow in a chemical vapor deposition (CVD) system. The films showed improved conductivity and transmittance, $600 \Omega / \square$ and $96.5 \%$ at $500 \mathrm{~nm}$, respectively [13]. For touch sensor applications, it has a sheet resistance less than $100 \Omega / \square$ and optical transparency $~ 90 \%$. In this study, we synthesize uniformly distributed graphene domains on standing copper foil in a low-pressure chemical vapor deposition (LPCVD) system. 


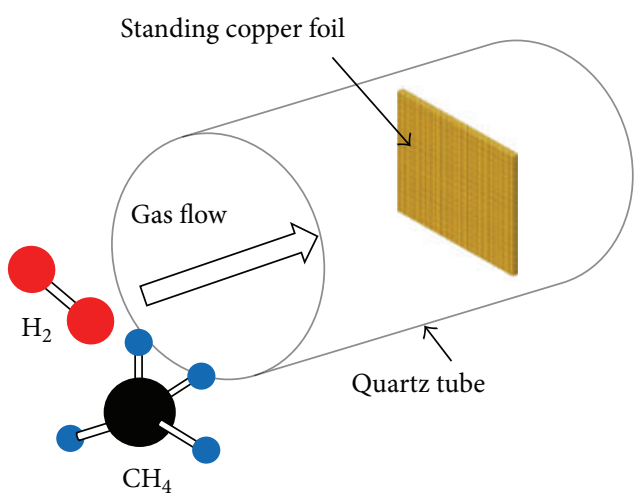

(a)

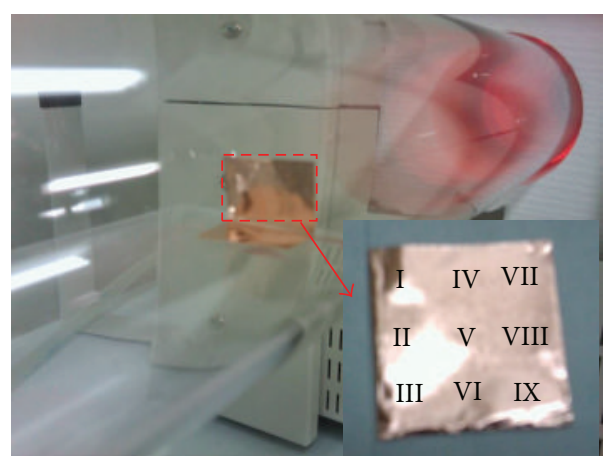

(b)

FIGURE 1: (a) Schematic representation of the LPCVD system showing the (b) copper foil standing on the quartz plate. The inserted image displays marks on the copper foil for SEM measurement.
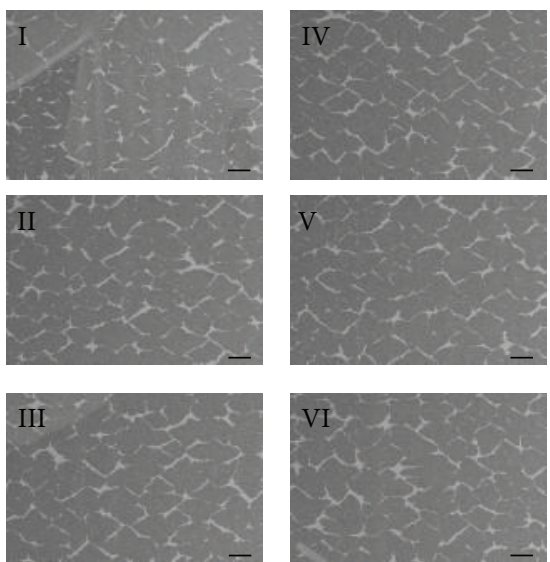

(a) Standing copper method
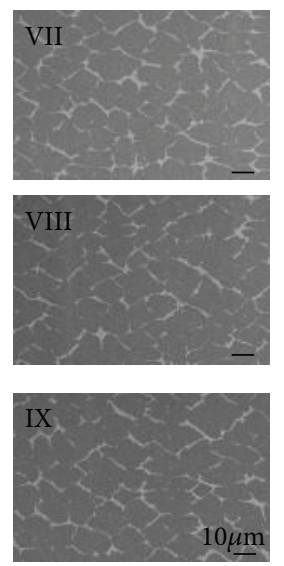

um

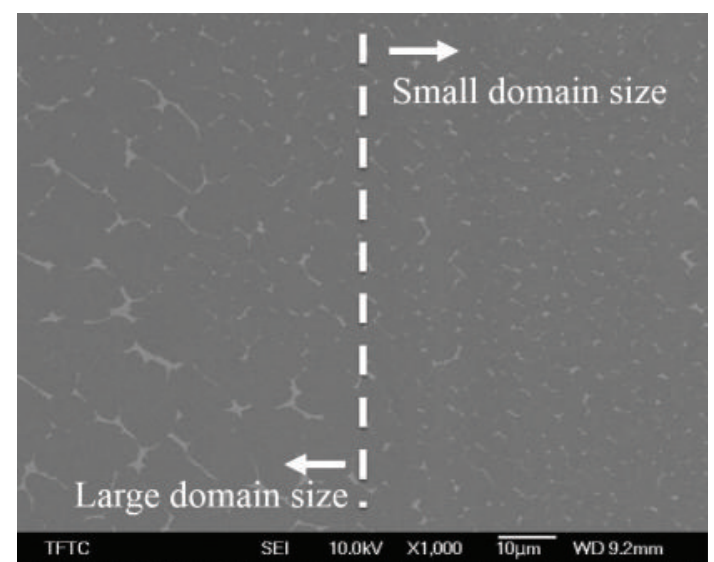

(b) Conventional method

FIGURE 2: (a) SEM images show the distribution of graphene domains on the standing copper foil (the scale bars are $10 \mu \mathrm{m}$ ). (b) SEM image shows the poor distribution of graphene domains on copper foil with conventional method.

\section{Experiments}

Graphene films were grown on $25 \mu \mathrm{m}$ thick copper foil (99.8\%, Alfa-Aesar, item no. 13382) using an LPCVD method similar to that used in a previous study [14]. The copper was electropolished in a homemade electrochemistry bath. The electropolishing solution was made up of $50 \mathrm{~mL}$ of deionized (DI) water, $20 \mathrm{~mL}$ of ethanol, $20 \mathrm{~mL}$ of isopropyl alcohol (IPA), and $100 \mathrm{~mL}$ of phosphoric acid, and a voltage in the range from 2 to $4 \mathrm{~V}$ was applied for $60 \mathrm{~s}$. After this, the copper foil was washed with DI water and dried with nitrogen gas for $5 \mathrm{~min}$. In the growth process, a $2 \times 2 \mathrm{~cm}^{2}$ piece of folded copper foil was loaded into a 3 -inch reaction chamber. The chamber was pumped down to $10^{-3}$ torr, and then high purity hydrogen gas was introduced with a $20 \mathrm{sccm}$ of flow rate during temperature ramp-up of the furnace with 480 mTorr. The copper foil was annealed at $1035^{\circ} \mathrm{C}$ in the hydrogen flow for $40 \mathrm{~min}$. Then, $0.36 \mathrm{sccm}$ of methane (purity, 99.99\%) was used as a carbon source for another $3 \mathrm{~min}$ prior to observation of the distribution of each graphene domains. The working pressure was 500 mTorr. After the growth process, a rapid cool down method was applied. Polymethyl methacrylate (PMMA) was used to transfer graphene films by using spincoating method with 3000 r.p.m. for 20 s, then baking at $130^{\circ} \mathrm{C}$ for $1 \mathrm{~min}$ on a hot plate. Since graphene grows on both sides of the $\mathrm{Cu}$ foil, $\mathrm{O}_{2}$ plasma was applied to remove the unwanted graphene for $10 \mathrm{~s}$. The $\mathrm{Cu}$ foil was etched away by $\mathrm{FeCl}_{3}$ solution overnight. The PMMA/graphene stack was rinsed with DI water, $2 \%$ hydrochloric acid $(\mathrm{HCl})$, and DI water again. PMMA/graphene stacks were placed on the substrate, and the PMMA was then dissolved in an acetone bath over 24 hours. The distribution of the graphene domains was measured by scanning electron microscopy (SEM); the transmittance spectra of the continuous grapheme 


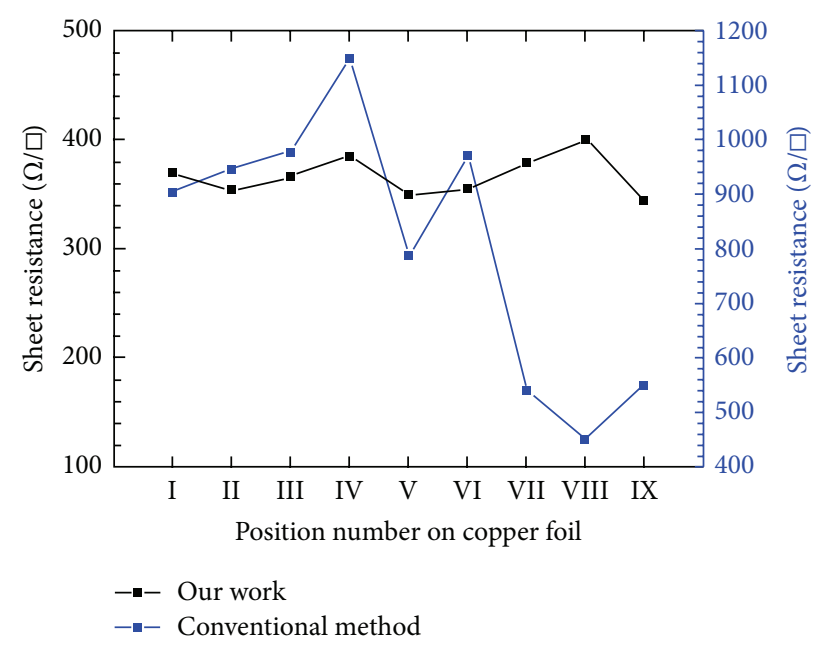

FIgURE 3: The comparison of the standing copper method and the conventional method on the electrical property.

films were measured by a Hitachi U-4100 spectroscope; the sheet resistance was measured using a four-probe stage; and the quality of graphene film was measured by Raman spectroscopy with an excitation laser beam at a wavelength of $514 \mathrm{~nm}$. The laser was focused by a 50x objective lens with a numerical aperture value of 0.75 onto about a $1 \mu \mathrm{m}^{2}$ area of the sample, and the laser power at the focused spot was $2 \mathrm{~mW}$ for the $514 \mathrm{~nm}$ wavelength.

\section{Results}

Figure 1(a) shows a schematic representation of the LPCVD system. The copper foil was folded and standing on the quartz plate, as shown in Figure 1(b). The image inset in Figure 1(b) shows marks on the standing copper for SEM measurement after the growth process. The distribution of the graphene domains has been a problem in previous studies [10-12]. However, we were able to grow a uniformly distributed graphene domain on the standing copper. The carbon atoms were easily transported to the copper surface, and the density of the graphene domain was controlled by the hydrogen flow rate and working pressure [11]. Figure 2(a) shows the average size of the graphene domain, which was about $\sim 20 \mu \mathrm{m}$ with $\sim 90 \%$ coverage area in our work. Nevertheless, Figure 2(b) shows the poor distribution of graphene domain with conventional way and the setups similar to one described elsewhere [14]. These results indicate that using the standing copper method can improve the distribution of the graphene domains at different positions on the same copper foil along the forward direction of the gas flow. We compare the sheet resistance between our case and the conventional method after graphene film transferred on glass substrates. Figure 3 demonstrates that our work results in a uniform performance on the electrical property, but the conventional synthesis method for graphene film results in a widening gap of performance on the electrical property, and this is a significant issue for many applications. Figure 4(a) displays the Raman spectrum whose peaks are typical of graphene, including a $2 \mathrm{D} / \mathrm{G}$ ratio as high as $\sim 2.6$. The full width half maximum (FWHM) of the $2 \mathrm{D}$ peak is $28.5 \mathrm{~cm}^{-1}$; the existence of single layer graphene was confirmed. A small $\mathrm{D} / \mathrm{G}$ peak ratio of 0.22 indicates that the amount of defects introduced during the transfer process was negligible. The inserted image in Figure 4(a) shows a $1 \times 1 \mathrm{~cm}^{2}$ sized piece of graphene film that was transferred onto a $\mathrm{SiO}_{2}(300 \mathrm{~nm}) / \mathrm{Si}$ wafer for the Raman measurement. The transmittance data as a function of wavelength for the graphene stacks is shown in Figure 4(b). The graphene films placed on a PET substrate ranged from single layer to four layers with a relatively high transmittance of about $88.86 \%$ at $\lambda=550 \mathrm{~nm}$ wavelength. The attenuation coefficient ( $\alpha=2.65 \%$ ) was fitted by Beer's Law. This is much closer to the theoretical value of $2.3 \%$ at the $\lambda=550 \mathrm{~nm}$ wavelength [15]. The higher attenuation for this result was perhaps due to the metal clusters, metal ions, and wrinkles from the transfer process. Figure 4(c) shows the sheet resistance of the monolayer graphene obtained with the standing copper method which was $359 \Omega / \square$. The value was $178 \Omega / \square$ for four layers. It was easy to fabricate heavily p-doped graphene with $63 \% \mathrm{HNO}_{3}$ for $5 \mathrm{~min}$ [9], after which the sheet resistance of the four-layer graphene film decreased from $178 \Omega / \square$ to $84.6 \Omega / \square$. The results showed an obvious decrease in the average sheet resistance. Based on this achievement, we further studied the effect of bending on the sheet resistance by fabricating a large scale graphene $\left(5 \times 5 \mathrm{~cm}^{2}\right)$ transparent conductive electrode (TCE) on a PET substrate (Figure 4(d) inset), as shown in Figure 4(d). The graphene TCE was subjected to a two-point bending stage. The sheet resistance increased from $0.1 \%$ to $9.8 \%$ with a bending radius from $10 \mathrm{~mm}$ to $4 \mathrm{~mm}$, respectively. After the bending radius reached $4 \mathrm{~mm}$, the sheet resistance increased by only $0.9 \%$ and showed faultless recovery after straightening.

\section{Conclusion}

In summary, a method for the growth of uniformly distributed graphene domains on a standing copper substrate via low-pressure chemical vapor deposition has been proposed. The values of the sheet resistance of four-layer graphene before and after being doped by $\mathrm{HNO}_{3}$ were $178 \Omega / \square$ and $84.6 \Omega / \square$, respectively. The transmittance data as a function of the wavelength for the four-layer graphene shows values as high as $88.86 \%$ at the $\lambda=550 \mathrm{~nm}$ wavelength. The standing copper method can be scaled up provided there are no limitations of the substrate and growth system. The uniformity of the sheet resistance is strongly dependent on the distribution of the graphene domains. Owning to the diffusion of carbon, either on the standing copper foil was the same. This method improved the distribution of the graphene domains at different positions on the same piece of copper foil along the forward direction of the gas flow. We believe this method can be a candidate for some transparent conductive electrode applications. 


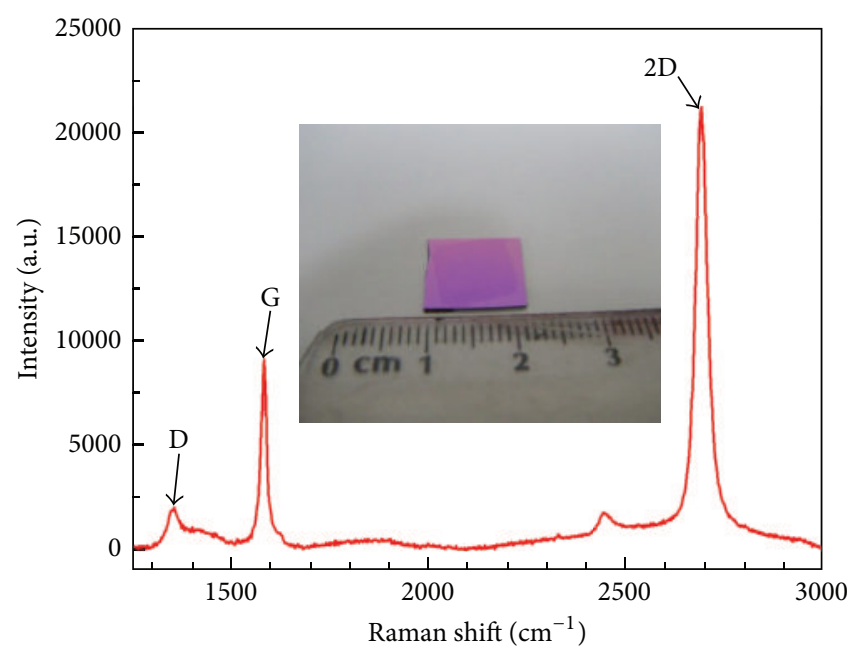

(a)

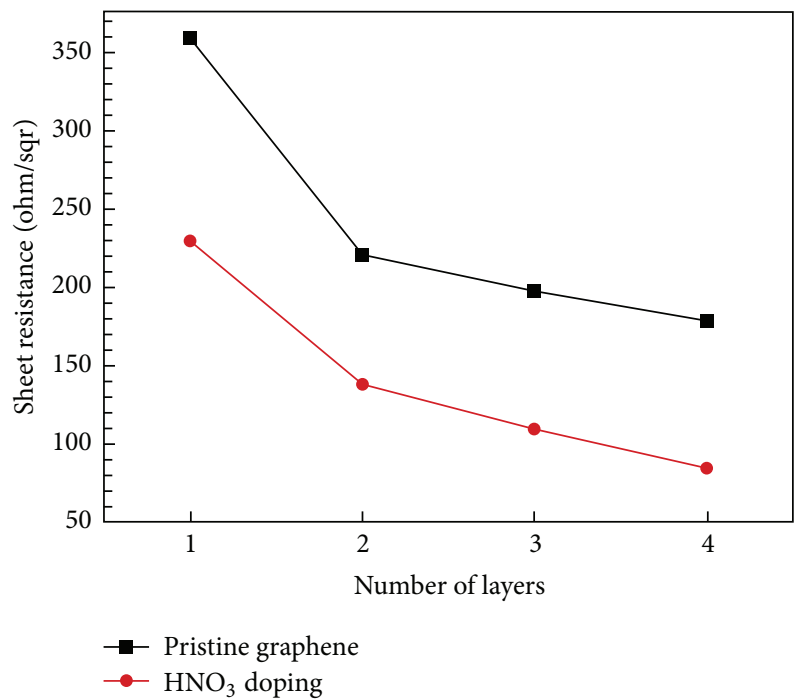

(c)

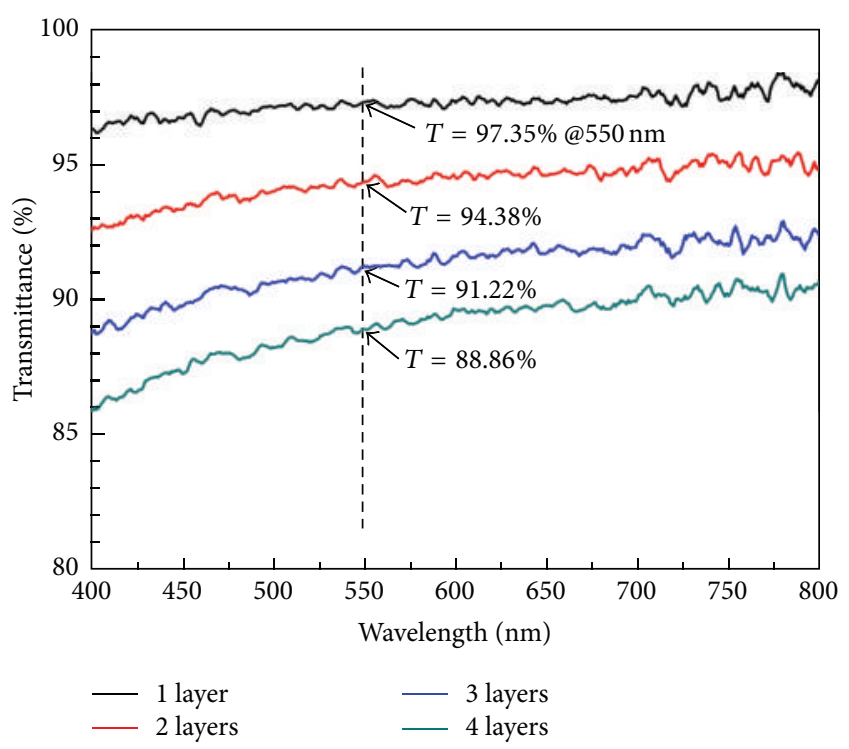

(b)

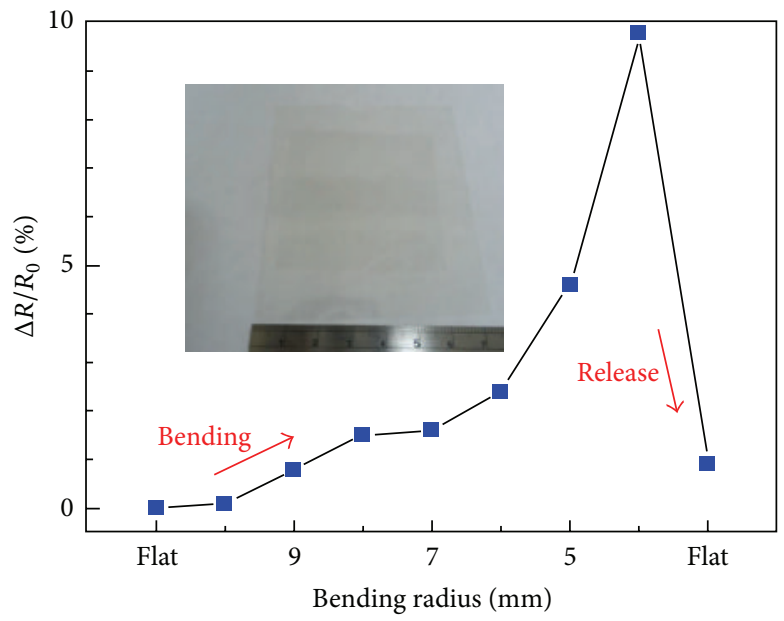

(d)

FIGURE 4: (a) Raman spectrum of monolayer graphene on the $\mathrm{SiO}_{2}(300 \mathrm{~nm}) / \mathrm{Si}$ wafer (inserted image). The 2D/G ratio is about $\sim 2.6$, and the FWHM of the $2 \mathrm{D}$ peak is $28.5 \mathrm{~cm}^{-1}$. A small D/G peak ratio of 0.22 was introduced during the transfer process. (b) Transmittance of stacked graphene films at $\lambda=550 \mathrm{~nm}$ as a function of the number of stacked graphene layers. (c) Sheet resistance of stacked graphene films as a function of the number of stacked graphene layers before and after being doped by $\mathrm{HNO}_{3}$. (d) Variation in the electrical resistance of the graphene film for a bending radius from $10 \mathrm{~mm}$ to $4 \mathrm{~mm}$.

\section{Acknowledgment}

The authors would like to thank the National Science Council of the Republic of China, Taiwan, for financially supporting this research under Contract no. NSC 102-ET-E-008-002-ET.

\section{References}

[1] E. Tsuji, N. Hirata, Y. Aoki, and H. Habazaki, "Preparation of non-annealed anatase $\mathrm{TiO}_{2}$ film on ITO substrate by anodizing in hot phosphate/glycerol electrolyte for dye-sensitized solar cells," Materials Letters, vol. 91, pp. 39-41, 2013.
[2] Y. C. Lin, S. J. Chang, Y. K. Su et al., "InGaN/GaN light emitting diodes with $\mathrm{Ni} / \mathrm{Au}, \mathrm{Ni} / \mathrm{ITO}$ and ITO P-type contacts," SolidState Electronics, vol. 47, no. 5, pp. 849-853, 2003.

[3] M. H. Ahn, E.-S. Cho, and S. J. Kwon, "Effect of the duty ratio on the indium tin oxide (ITO) film deposited by in-line pulsed DC magnetron sputtering method for resistive touch panel," Applied Surface Science, vol. 258, no. 3, pp. 1242-1248, 2011.

[4] V. C. Tung, L.-M. Chen, M. J. Allen et al., "Low-temperature solution processing of graphene-carbon nanotube hybrid materials for high-performance transparent conductors," Nano Letters, vol. 9, no. 5, pp. 1949-1955, 2009. 
[5] L. Li, K. S. Hui, K. N. Hui et al., "Synthesis and characterization of NiO-doped P-type AZO films fabricated by sol-gel method," Materials Letters, vol. 68, pp. 283-286, 2012.

[6] A. K. Geim, "Graphene prehistory," Physica Scripta, vol. 2012, Article ID 014003, 2012.

[7] Y. Zhu, Z. Sun, Z. Yan, Z. Jin, and J. M. Tour, "Rational design of hybrid graphene films for high-performance transparent electrodes," ACS Nano, vol. 5, no. 8, pp. 6472-6479, 2011.

[8] X. Li, Y. Zhu, W. Cai et al., "Transfer of large-area graphene films for high-performance transparent conductive electrodes," Nano Letters, vol. 9, no. 12, pp. 4359-4363, 2009.

[9] S. Bae, H. Kim, Y. Lee et al., "Roll-to-roll production of 30-inch graphene films for transparent electrodes," Nature Nanotechnology, vol. 5, no. 8, pp. 574-578, 2010.

[10] W. Wu, Q. Yu, P. Peng, Z. Liu, J. Bao, and S.-S. Pei, "Control of thickness uniformity and grain size in graphene films for transparent conductive electrodes," Nanotechnology, vol. 23, no. 3, Article ID 035603, 2012.

[11] Z. Yan, J. Lin, Z. W. Peng, Z. Z. Sun, Y. Zhu, and L. Li, “Toward the synthesis of wafer-scale single-crystal graphene on copper foils," ACS Nano, vol. 6, pp. 9110-9117, 2012.

[12] X. Li, C. W. Magnuson, A. Venugopal et al., "Large-area graphene single crystals grown by low-pressure chemical vapor deposition of methane on copper," Journal of the American Chemical Society, vol. 133, no. 9, pp. 2816-2819, 2011.

[13] J. Zhang, P. A. Hu, X. N. Wang, Z. L. Wang, D. Q. Liu, B. Yang et al., "CVD growth of large area and uniform graphene on tilted copper foil for high performance flexible transparent conductive film," Journal of Materials Chemistry, vol. 22, pp. 18283-18290, 2012.

[14] X. Li, W. Cai, J. An et al., "Large-area synthesis of high-quality and uniform graphene films on copper foils," Science, vol. 324, no. 5932, pp. 1312-1314, 2009.

[15] R. R. Nair, P. Blake, A. N. Grigorenko et al., "Fine structure constant defines visual transparency of graphene," Science, vol. 320 , no. 5881, p. $1308,2008$. 

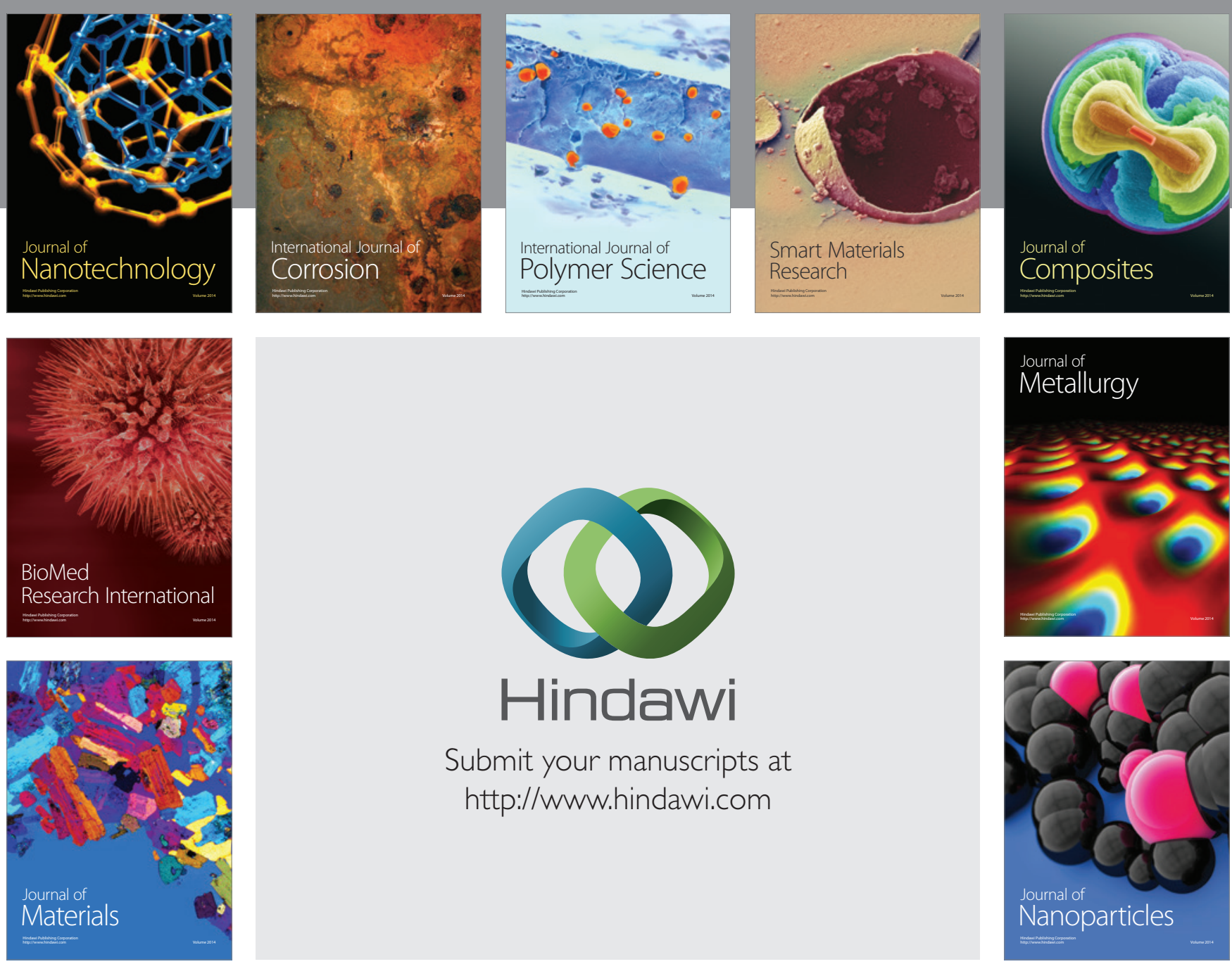

Submit your manuscripts at http://www.hindawi.com
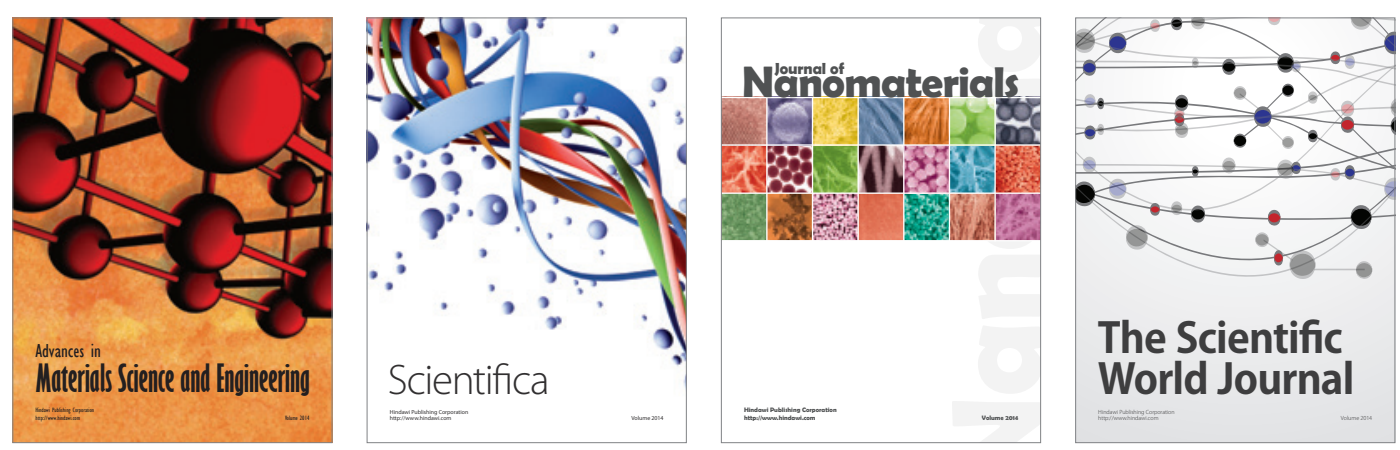

\section{The Scientific World Journal}
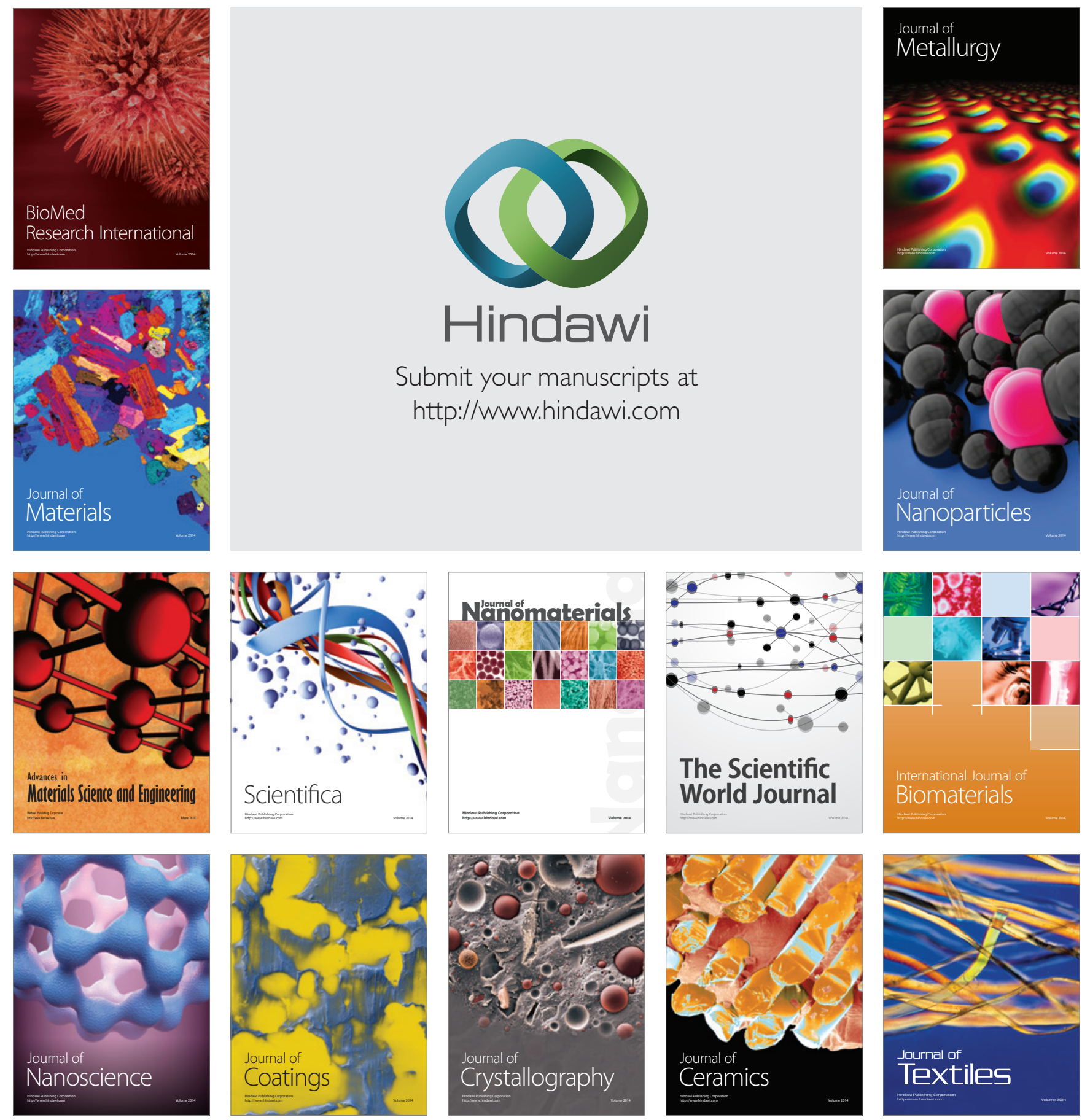